\title{
Decreased miR-17-92 Cluster Correlates With the Senescent Features and Disrupted Oxidative Homeostasis, and the Impaired Therapeutic Efficacy of Adipose Tissue-derived Mesenchymal Stem Cells
}

\section{Yelei Cen}

Zhejiang University First Affiliated Hospital State Key Laboratory for Diagnosis and Treatment of Infectious Diseases

Jinjin Qi

Zhejiang University First Affiliated Hospital State Key Laboratory for Diagnosis and Treatment of Infectious Diseases

\section{Liang Chen}

Zhejiang University School of Medicine First Affiliated Hospital

Yanning Liu ( $\square$ liuyanning@zju.edu.cn )

Zhejiang University First Affiliated Hospital State Key Laboratory for Diagnosis and Treatment of Infectious Diseases https://orcid.org/0000-0002-0554-6622

Guohua Lou

Zhejiang University First Affiliated Hospital State Key Laboratory for Diagnosis and Treatment of Infectious Diseases

\section{Research Article}

Keywords: Acute liver failure, Adipose tissue-derived mesenchymal stem cells, Senescence, MiR-17-92 cluster, Oxidative homeostasis

Posted Date: February 11th, 2022

DOI: https://doi.org/10.21203/rs.3.rs-1333637/v1

License: () (1) This work is licensed under a Creative Commons Attribution 4.0 International License. Read Full License 


\section{Abstract}

Background: Aging and replicative cellular senescence is associated with the reduced therapeutic potential of mesenchymal stem cells (MSCs) on variety of diseases. This study aimed to determine the mechanism in regulating MSC senescence and further explore a modification strategy to reverse the senescence-associated cell dysfunction for improving the therapeutic efficacy of MSCs on acute liver failure (ALF).

Methods: Adipose tissue-derived MSCs from old-aged mice (oAMSCs) or young mice were intravenously administrated into the mice with lipopolysaccharide and D-galactosamine (LPS/GalN)-exposure and their effects were evaluated by serumbiochemical and liver histological analysis. SA- $\beta$-gal staining, expression analysis on p21, p16 and senescence-associated secretory phenotype markers, and reactive oxygen species (ROS) generation assay were used for determine the senescent features in aged AMSCs and replicative senescent AMSCs. MiRNA expression assay, small interfering RNA and miRNA transfection, and thioredoxin (TRX) activity assay were performed for further elucidate the mechanisms in AMSC senescence. MiRNA-modified AMSCs were used for determine whether cellular miR-17-92 cluster level can as an index to evaluate the therapeutic potential of AMSCs on ALF.

Results: oAMSCs displayed senescent phenotypes and showed reduced therapeutic efficacy on LPS/GalN-induced ALF, as determined by reduced hepatic necrosis and serum liver function indicators, and inflammatory cytokine levels. Then we found that the miR-17-92 cluster, especially miR-17 and miR20a, was obviously decreased in oAMSCs and replicative senescent AMSCs. The decreased miR-17-92 level was consistent with the declined oncogene c-Myc level during AMSC senescence and may mediate c-Myc stemness addiction. Further experiments revealed that miR-17-92 could modulate AMSC senescence by affecting the expression of classic senescence protein p21 and also the oxidative homeostasis in AMSCs through TXNIP-mediated TRX activity regulation. Furthermore, modification of AMSCs with the above two key miRNAs in miR-17-92 cluster could reverse the senescent features of oAMSCs and remodeling the therapeutic potential of senescent AMSCs on ALF.

Conclusions: c-Myc-regulated miR-17-92 contributes to the increased p21 expression and dysregulated redox system during AMSC senescence. The cellular miR-17-92 cluster level can be used both as an index for evaluating and as a modification target for improving the therapeutic potential of AMSCs.

\section{Background}

Acute liver failure (ALF) is a life-threatening clinical syndrome characterized by rapid hepatocellular necrosis due to various acute injuries induced by hepatotoxic drugs, immune-mediated attack, or viral infections. Liver transplantation and artificial liver therapies are presented as the main clinical treatments for ALF [1]. However, the shortage of available donor livers limits the widespread clinical application of liver transplantation, and multiple postoperative complications also limit the efficacy of artificial liver therapy against ALF [2]. Increasing number of studies showed that mesenchymal stem cells (MSCs) can 
effectively treat ALF [3]. We have also determined the therapeutic efficacy of adipose tissue-derived mesenchymal stem cells (AMSCs) or AMSC-derived exosomes on various liver diseases including liver fibrosis, hepatocellular carcinoma, and $\operatorname{ALF}[4,5]$. However, there are still some issues should be addressed before clinical application of MSCs on ALF, especially in the context of elderly donors. Several studies have shown that a decrease in the in vitro proliferation capacity and multidirectional differentiation potential of human bone marrow-derived MSCs with the increase in the age of the donors [6]. In addition, AMSCs from aged donors [7], as well as from individuals suffering from metabolic disorders [8] showed a diminished regenerative potential. Further study showed that MSCs isolated from aged donors exhibit senescent features, resulting in more senescence-associated $\beta$-galactosidase (SA- $\beta$ gal)-positive cells and increased secretion of senescence-associated secretory phenotype (SASP) cytokines [9].

Although many studies have been shown the aging-associated cell senescence of MSCs and reported the reduced therapeutic potential of AMSCs from aged donors, the exact mechanism underlying the agingassociated cell dysfunction and therapeutic role of aged AMSCs on ALF remains unclear. In the current study, we compared the therapeutic efficacy of AMSCs from aged mice to those from young mice on ALF, and then investigate the potential mechanisms contributed to the aging-associated cell senescence of AMSCs. Finally, we explored the modification strategy to reverse the aging of AMSCs. Our study may provide an indicator for evaluating the AMSC quality for clinical usage and also provide a promising approach for improve the therapeutic efficacy AMSCs on ALF.

\section{Methods}

\section{Isolation and identification of AMSCs}

C57BL/6J mice were purchased from Nanjing BioMedical Research Institute of Nanjing University. The experimental procedures were reviewed and approved by the Institutional Animal Care and Use Committee in our institution. Inguinal adipose tissues were obtained from male mice (8 weeks old as young mice, 20 months old as aged mice). Adipose tissues were digested with $0.075 \%$ collagenase type I (Sigma-Aldrich, St Louis, MO, USA) for 30 min, then centrifuged and washed with PBS for 2 times. Finally, the isolated cells were cultured in murine MesenCult ${ }^{\mathrm{TM}}$ Expansion Kit (Stemcell) containing $2 \mathrm{mM} \mathrm{L-}$ glutamine (Thermo Fisher Scientific) and 1\% antibiotic-antimycotic. Cells were maintained and expanded by 2-10 passages before usage.

\section{ALF mouse model and AMSCs treatment}

C57BL/6J Mice (aged 5-6 weeks) were intraperitoneally injected with Lipopolysaccharide (LPS, 10 $\mu \mathrm{g} / \mathrm{kg}$, Sigma-Aldrich) and D-galactosamine (GalN, $400 \mathrm{mg} / \mathrm{kg}$, Sigma-Aldrich) to establish a mouse model of ALF. AMSCs from young mice (yAMSC) or aged mice (oAMSC), P2, P6 or P10 AMSCs from a culture-induced senescent cell model, and miR-17 or miR-20a-modified oAMSCs (oAMSC-M17, oAMSCM20a) or control miRNA-modified oAMSCs (oAMSC-M67) were administered via the tail vein $\left(2 \times 10^{5}, \mathrm{n}=\right.$ 6) immediately after LPS/GaIN injection. The control group was administered with vehicle alone $(n=6)$. 
At $6 \mathrm{~h}$ after LPS/GalN injection, the mice were sacrificed, and serum and liver samples were collected. Serum was evaluated for biochemical parameters. The liver samples were evaluated for histochemistry and Western blot analysis.

\section{Isolation and detection of miRNA}

Total RNA enriched with miRNAs was isolated from yAMSCs, oAMSCs and different passaged AMSCs by using a miRVana miRNA isolation kit (Thermo Fisher) according to the manufacturer's instructions. Complementary DNA was synthesized from the isolated miRNAs by using TaqMan ${ }^{\text {TM }}$ mmu-miRNA-speci $\mathrm{C}$ primers including miR-17-19 cluster (Thermo Fisher) and TaqMan ${ }^{\mathrm{TM}}$ MicroRNA Reverse Transcription Kit (Thermo Fisher). Real-time PCR was then performed following the manufacturer's instructions (Thermo Fisher) to examine the expression of miR-17-19 cluster, especially miR-17 and miR-20a. Data were normalized over the average cycle threshold (CT) value of U6, and the $2^{-\triangle \Delta C T}$ method was used to determine the relative miRNA expression.

\section{RNA isolation and qPCR}

Total RNA was isolated from AMSCs by using TRIzol. First-strand CDNA was subsequently synthesized followed by qPCR analysis by using ABI Prism 7900 (Applied Biosystems) to examine the expression levels of TNF- , IL-6, MCP1, PAI-1, Mmp3 and Mmp13, $\beta$-actin were used as internal controls. The $2^{-\triangle \triangle C T}$ method was used to determine the relative mRNA expression levels of these genes.

\section{Liver histological and serum biochemical analysis}

Liver tissues were processed for paraffin embedding and were sectioned into $4 \mu \mathrm{m}$ sections. The sections were then routinely stained with hematoxylin and eosin (H\&E staining). The serum levels of alanine aminotransferase aspartate (ALT) and aspartate aminotransferase (AST) were analyzed by standard analyzer DRICHEM 4000ie (FUJIFILM). Total bilirubin (TBiL) was measured using standard clinical chemistry techniques (Integra II; Roche, UK).

\section{Cytokine detection by ELISA}

The murine serum levels of the cytokines including TNF-a, IL-6, and MCP-1 (MultiSciences, Shanghai, China) were assayed by commercial ELISA kits following the manufacturer's instructions.

\section{SA- $\beta$-gal staining}

SA- $\beta$-gal staining was used to determine cell senescence of AMSCs. Briefly, yAMSCs, oAMSCs and different passaged AMSCs were seeded at a density of $5 \times 10^{4} /$ well in a 6-well plate with complete medium and incubated for $24 \mathrm{~h}$ at $37^{\circ} \mathrm{C}$ in $5 \% \mathrm{CO}_{2}$. Afterwards, the cells were fixed with $4 \%$ paraformaldehyde (PFA, Sigma-Aldrich, USA) at room temperature for $20 \mathrm{~min}$ and subsequently stained by SA- $\beta$-gal staining kit (Beyotime, Shanghai, China) following the manufacturer's instructions. Positive 
senescent cells stained in blue were observed using an inverted microscope (AxioCam ERc 5s, Carl Zeiss, Germany).

\section{Western blot analysis}

AMSCs were lysed in ice-cold RIPA buffer (Beyotime Biotechnology, Jiangsu, China) containing 1\% protease inhibitors (Pierce) for $30 \mathrm{~min}$ at $4^{\circ} \mathrm{C}$. Lysates $(25 \mu \mathrm{g}$ of protein) were analyzed by $10 \%$ sodiumdodecyl sulfate-polyacrylamide gel electrophoresis (Sigma-Aldrich) and transferred to nitrocellulose membranes (Merck Millipore, Billerica, MA, USA) according to standard procedures. Primary antibodies were used as follows: p21 (1:1000, Abcam), p16 (1:2000, Abcam), TXNIP (1:2000, Abcam), TRX (1:1000, Cell Signaling Technology), c-Myc (1:1000, Abcam), and GAPDH (1:3000, HuaBio). Protein bands were developed using the enhanced chemiluminescence $(E C L)$ system and were visualized using the ChemiScope Western Blot Imaging System (Clinx Science Instruments Co., Ltd). The gray value assay was performed by using Image J software (Rawak Software, Inc. Germany).

\section{Determination of reactive oxygen species (ROS) generation}

AMSCs were stained with $5 \mu \mathrm{M}$ H2DCF-DA (Life Technologies) for 10 min and then washed twice with ice-cold PBS and ROS generation was determined by FACS analysis. ROS generation was quantified as the increase in mean fluorescence intensity (MFI).

\section{Transfection of small interfering RNA (siRNA) and miRNA}

SiRNA against c-Myc, TXNIP and scrambled siRNA were purchased from Guangzhou RiboBio Co., Ltd. and miR-17 and miR-20a mimics, miRNA mimic negative control (M-Ctrl), miR-17 and miR-20a inhibitors, and miRNA inhibitor negative control (I-Ctrl) were also purchased from RiboBio. RNAiMAX (Thermo Fisher Scientific) was used for siRNA and miRNA transfection. At 75\% confluence, AMSCs were transfected with siRNA $(100 \mathrm{~nm})$ and/or miRNA $(50 \mathrm{nM})$ at $37^{\circ} \mathrm{C}$. At $48 \mathrm{~h}$ after transfection, the cells were harvested for qPCR and Western blot analysis.

\section{Thioredoxin (TRX) activity assay}

TRX activity was measured by the Thioredoxin Fluorometric Activity Assay Kit (Cayman, Item No.500228) according to the manufacturer's instruction. Briefly, $0.5-1 \mathrm{mg} / \mathrm{ml}$ of cell lysates were prepared in cold buffer (100 mM Tris-HCl pH 7.5, 1 mM EDTA), supplemented with protease inhibitor. Then $20 \mu \mathrm{l}$ cell lysates were mixed with $10 \mu \mathrm{l}$ TRX reductase, $10 \mu \mathrm{lNADPH}$ and $40 \mu \mathrm{l}$ assay buffer. After the reaction was performed at $37^{\circ} \mathrm{C}$ for $30 \mathrm{~min}, 20 \mu \mathrm{l}$ reconstituted Eosin-Labeled Insulin was added. Measure the fluorescence at excitation and emission wavelength of $520 \mathrm{~nm}$ and $560 \mathrm{~nm}$, once every minute for $60 \mathrm{~min}$ by Synergy Neo2 (BioTek).

\section{Construction of miRNA-modified AMSCs}


The miR-17 (LV-miR-17) and miR-20a (LV-miR-20a) expression vectors and the control vector cel-miR-67 (LV-cel-miR-67) were constructed by cloning their precursor sequences, together with a short upstream

and a short downstream flanking region, into lentiviral expression vectors. Before infection, $1 \times 10^{6}$ AMSCs were seeded in $10 \mathrm{ml}$ complete medium overnight and then transfected with $100 \mathrm{nM} \mathrm{LV}-\mathrm{miR}-17$, LV-miR-20a or LV-cel-miR-67 at a multiplicity of infection of 10 in the presence of polybrene $(8 \mu \mathrm{g} / \mathrm{ml}$; Sigma-Aldrich, USA) for $24 \mathrm{~h}$. Stable transfectants were used in the subsequent experiments.

\section{Statistics}

Statistical evaluation was performed using independent samples t-tests between two groups and using one-way analysis of variance with Tukey's post hoc test between three or more groups. All data are presented as the mean \pm SD. $P<0.05$ was considered significant.

\section{Results}

\section{AMSCs from aged mice show an abated protective effect on acute liver failure}

We firstly determined the therapeutic efficacy of AMSCs in LPS/GalN-induced ALF mouse model. As shown in Figure 1A and B, LPS/GalN induced significant liver injury, as indicated by dramatically elevated serum ALT, AST, and TBiL levels accompanied by massive hepatic necrosis, while administration of the AMSCs from young mice (yAMSC) resulted in a remarkable reduction in serum ALT, AST, and TBiL levels (Fig. 1A) and less hepatic necrosis (Fig. 1B). The elevated serum levels of inflammatory cytokines, such as TNF-a, IL-6, and MCP-1 (Fig. 1C), in LPS/GalN-injected mice were also significantly reduced by yAMSCs administration compared with vehicle-treated mice at $6 \mathrm{~h}$ after LPS/GalN injection. However, administration of the AMSCs from old-aged mice (OAMSC) showed an abated protective effect on ALF as compared to yAMSCs administration (Fig. 1A-C). These data indicate that aging-related change of cell characteristics may affect the therapeutic efficiency of AMSCs on ALF.

\section{AMSCs from aged mice show senescent features}

To determine whether the different efficiency of AMSCs on ALF related to stem cell aging, we analyzed the morphology and cell senescent features of yAMSCs and oAMSCs at passage 2. YAMSCs displayed a characteristic spindle-like morphology, whereas oAMSCs were larger and irregular (Fig. 2A). There were more SA- $\beta$-gal-stained cells in cultured oAMSCs as compared to yAMSCs (Fig. 2B). Moreover, CCK-8 assay showed that the proliferation capacity of oAMSCs was significantly lower than that of yAMSCs (Fig. 2C). Furthermore, the protein levels of cell senescent molecular markers, p16 and p21, in cultured oAMSCs were significantly increased than that in yAMSCs (Fig. 1D). The mRNA levels of SASP markers including Mmp3, Mmp13, PAI-1, MCP-1, TNF- , and IL-6 were also remarkably increased in oAMSCs than that in yAMSCs (Fig. 1E). These data demonstrated that the AMSCs from aged mice displayed a senescent phenotype, compared to the AMSCs from young mice.

MiR-17-92 cluster associates with the cellular senescence of AMSC by targeting p21 
MiRNA has been found to play critical roles in many age-related diseases, including cardiovascular and neurological diseases [10]. Differentially expressed miRNAs in cellular senescence has already been reported in cultured fibroblasts [11]. However, their role in MSC senescence and aging has not been fully characterized. Since among the reported cell senescence-related miRNAs, miR-17, 18a, 19a, 19b-1, 20a, and 92a-1, are all derived from one miRNA cluster, the miR-17-92 cluster (Fig. 3A), we then examined whether this miRNA cluster is related to AMSC senescence. RT-qPCR analysis confirmed that the expression levels of the miR-17-92 cluster miRNAs, especially miR-17 and miR-20a, were obviously decreased in oAMSCs compared to that in yAMSCs (Fig. 3B). The downregulated levels of miR-17 and miR-20a were also observed in the culture-induced senescence of AMSCs by comparing their levels between the passage 2 (P2) and the P7 of AMSCs (Fig. 3C). We next investigated whether the miR-17 and miR-20a by themselves were sufficient for overcoming senescence of AMSC. The proliferation capacities of yAMSCs transduced with miR-17 or miR-20a inhibitors were significantly decreased as compared to those transduced with control inhibitors (Fig. 3D). While, the accumulation of SA- $\beta$-gal in the miR-17 or miR-20a inhibitor-transfected yAMSCs were markedly increased (Fig. 3E). These data indicate that the decreased miR-17-92 cluster maybe related to the cellular senescence of AMSCs and miR-17 and miR-20a are both necessary and sufficient for affecting AMSC senescence, and thus are the key components mediating the anti-senescence activity of miR-17-92. Since the miR-17-92 cluster is recognized as cell cycle regulators via direct targeting of p21 [12], encoded by the CDKN1A gene (Fig. 3F), we then investigated the regulation of miR-17 and miR-20a on p21 by the transfection with their mimics or inhibitors. As anticipated, overexpression of miR-17 and miR-20a by transfection with their mimics both reduced p21 protein levels in oAMSCs (Fig. 3G). On the contrary, by transfection with miR-17 and miR-20a inhibitors, the p21 protein levels were increased accordingly in yAMSC (Fig. $3 \mathrm{H})$. These data strongly indicate that the upregulated expression of p21 in senescent AMSCs may be caused by the reduced expression of miR-17-92 cluster.

\section{c-Myc-regulated miR-17-92 is involved in the senescence of AMSC}

It is known that miR-17-92 can be directly trans-activated by c-Myc, which is related to the stemness of variety of cells [13]. We therefore hypothesized that c-Myc-regulated miR-17-92 maybe involved in the senescence of AMSC. As anticipated, the expression level of c-Myc was higher in yAMSC than that in oAMSCs and was also remarkably decreased from P2 to P7, a culture-induced senescent cell model of AMSCs (Fig. 4A). The decreased c-Myc level was consistent with the downregulated miR-17-92 level during cellular senescence. To further consolidate the role of c-Myc in controlling miR-17-92, we specifically inhibited c-Myc expression by siRNA in AMSCs (Fig. 4B). We found that down-regulation of cMyc reduced miR-17 and miR-20a levels whereas increased p21 level and SA- $\beta$-gal accumulation in AMSCs and subsequently reduced the proliferation capacity of AMSCs (Fig. 4B-D). In addition, by further transfection of miR-17 or miR-20a mimics into AMSCs, we found that c-Myc siRNA-induced upregulation

of p21 and -reduced proliferation capacity could be markedly reversed by increasing in miR-17 or miR-20a levels (Fig. 4E and F). These observations indicated that c-Myc-induced miR-17-92 cluster plays a dominant role in the senescence of AMSC by regulating $\mathrm{p} 21$. 


\section{Decreased miR-17-92 cluster associates with the dysregulated redox system in senescent AMSCs}

Disrupted oxidative homeostasis has been characterized in senescent MSCs. High ROS level is responsible for age-related loss of cellular functions and also represents an important cause of cellular senescence [14]. We found that the ROS level was higher in oAMSCs than that in yMSCs and was also increased in the AMSCs from P2 to P7 (Fig. 5A). Redox regulation by TRX plays a crucial role in responses to oxidative stress [15]. As shown in Fig. 5B, the expression lever of TRX showed no obvious difference between oAMSCs and yAMSCs or between P2 and P7 AMSCs, whereas the activity of TRX was markedly decreased in the aging or replicative senescent AMSCs (Fig. 5C).

Our previous study showed that miR-17 could suppress the expression of thioredoxin-interacting protein (TXNIP) by directly targeting its mRNA 3' UTR (Fig. 5D). Since TXNIP is known as a negative regulator of TRX [15], we guessed the abnormal redox status and the decreased TRX activity in senescent AMSCs may due to the disrupted suppression of TXNIP by miR-17, which was declined in senescent AMSCs. As expected, TXNIP expression was upregulated in oAMSCs and P7 AMSCs compared to that in yAMSCs and P2 AMSCs, respectively (Fig. 5B). In addition, reduce miR-17 and miR-20a levels by transfection with their inhibitors could upregulate TXNIP expression consistent with the reduction of TRX activity in AMSCs, whereas miR-17 and miR-20a mimic transfection could downregulate TXNIP expression consistent with an increase in TRX activity in AMSCs (Fig. 5E and F). To further determine the role of TXNIP in regulating the oxidative homeostasis of AMSCs, we transfected a siRNA against TXNIP into oAMSCs and then examined the ROS level and the TRX activity. TXNIP knock-down partly reversed the increased ROS level and restored the TRX activity in OAMSCS, further substantiating a role for TXNIP in redox regulation (Fig. 5G-I). Moreover, TXNIP knock-down also reversed the decreased TRX activity and the enhanced ROS level in AMSCs by miR-17 or miR-20a inhibitor transfection (Fig. 5J and K). This clearly indicates that the dysregulated redox system in senescent AMSCs may due to the abnormal expression of TRX inhibitor TXNIP, which is negatively regulated by miR-17-92 cluster.

\section{MiR-17 and miR-20a levels correlated with the therapeutic efficacy of AMSCs on acute liver failure}

The above studies have shown that miR-17-92 cluster, especially miR-17 and miR-20a, is associated with AMSC senescence. We then determine whether the reduced therapeutic efficacy of senescent AMSCs on ALF was correlated with the decreased miR-17-92 level. The miR-17-92 cluster level of AMSCs was detected at passage 2, 6, and 10 by qPCR. As anticipated, the miR-17 and miR-20a levels were gradually declined from P2 to P10 (Fig. 6A). Administration of P2 AMSCs had better protective effects on LPS/GalN-induced ALF as compared to P6 AMSCs-treated mice but administration of P10 AMSCs, no protection against liver injury were evident as determined by serum ALT, AST and TBiL levels (Fig. 6B) and H\&E staining (Fig. 6C).

To further determine whether miR-17-92 cluster, especially miR-17 and miR-20a, modification can improve senescent AMSCs therapeutic efficacy on ALF, we constructed miR-17- or miR-20a-modified AMSCs by infection of LV-miR-17 (oAMSC-M17) and LV-miR-20a into oAMSCs (oAMSC-M20a). oAMSCs infection with LV-cel-miR-67, which has no known mRNA binding targets in mouse, was served as control (oAMSC- 
M67). As shown in Fig. 6E and F, the pathologic alteration and the upregulated serum ALT, AST, and TBIL induced by LPS/GalN could more effectively attenuated by oAMSC-M17 or oAMSC-M20a administration compare to oAMSC-M67 administration. These data suggest that the declined miR-17-92 cluster level may be correlated with the diminished therapeutic efficacy of senescent AMSCs on ALF and modification with miRNAs belong to this cluster may act as a strategy to enhance the therapeutic potential of AMSCs.

\section{Discussion}

Recently, MSCs are considered to be a highly attractive therapeutic strategy on variety of liver diseases including ALF, whereas, MSCs isolated from aged individuals exhibit a reduced proliferative activity and differentiation potential, as well as senescent features, limits their use in autologous transplantation [16]. In addition, in vitro expansion of primary MSCs for enough transplantation cells may also leads to cellular replicative senescence and loss of multipotency [17]. The altered protein expression of senescent MSCs is well studied and characterized. While, the role of miRNAs in MSC senescence and aging have not been fully characterized. In the present study, we showed that the therapeutic efficacy of AMSCs on ALF was markedly reduced in the AMSCs from old-aged mice or high passage, which exhibiting senescent features. Then we found that the decrease in miR-17-92 cluster levels may be associated with AMSC senescence by affecting the expression of classic senescence protein and oxidative homeostasis in AMSCs. Furthermore, modification of AMSCs with miR-17 and miR-20a, the two key miRNAs in miR-1792 cluster, could reverse the senescent features of oAMSCs and also remodeling the therapeutic potential of senescent AMSCs on ALF.

Increasing studies showed that miRNAs are key regulators and reliable markers of cellular senescence $[10,18]$. Numerous dysregulated miRNA have been identified in senescent MSCs and known to correlate with many age-related diseases. For example, miR-486-5p was increased in aged MSCs and could inhibit osteogenic and adipogenic differentiation and induce senescence of MSCs by targeting SIRT1 [19]. In addition, the increased miR-335 in aged MSCs as well as in y-irradiation-induced senescent MSCs was known to effect MSC senescent features through inhibition of AP-1 activity [20]. In this study, we found that the miRNA expression patterns, including miR-17-92 cluster, between the AMSCs from old-aged mice or young mice were markedly different (data not shown). qPCR analysis further showed that the levels of miR-17 and miR-20a, both derived from the miR-17-92 cluster and belonging to the miR-17 family, were decreased more obviously in aged AMSCs and replicative senescent AMSCs. Thus, we choose miR-17-92 cluster, especially miR-17 and miR-20a, for further study. The miR-17-92 cluster has been firstly identified as an oncogenic miRNA cluster [21]. Overexpression of miR-17-92 has been detected in various types of human cancer and known to promote cell cycle progression and proliferation, inhibit apoptosis, and induce tumor angiogenesis [21]. Recently, miR-17-92 cluster is increasingly concerned in aging research and the decrease in miR-17 family has been observed in human aging and senescent MSCs. We further showed that declined miR-17-92 cluster level was contributed to the concomitant up-regulation of p21 during AMSC senescence. Thus, via direct targeting the key cell cycle protein $\mathrm{p} 21$, the miR-17-92 cluster seems to maintain cell proliferation and survival, which is correlated with the progenitor/regenerative state of AMSCs. 
We next investigated how the miR-17-92 cluster expression was regulated. The oncogene c-Myc, also known as a stemness gene, has previously been reported to regulate cell proliferation, survival, apoptosis, and senescence, several of the key phenotypes associated with the addiction of miR-17-92 cluster [13, 22]. We found that the decreased c-Myc level was consistent with the downregulated miR-17-92 level during AMSC senescence. Moreover, c-Myc knock down-induced upregulation of p21 and -reduced cellular proliferation could be markedly reversed by increasing in miR-17 or miR-20a levels in AMSCs. We therefore deduced that c-Myc-regulated miR-17-92 is involved in the regulation of p21 expression during AMSC senescence and miR-17 and miR-20a are the key miR-17-92 components for sufficient abrogation of c-Myc reduction-induced p21 expression.

In addition to the regulation of $\mathrm{p} 21$ expression in cellular senescence, miR-17-92 cluster has been known to affect senescence by regulating several other genes, such as Smurf1 and SOSC1 [23, 24]. Recently, disrupted oxidative homeostasis and high ROS level have been characterized in MSC senescence. TXNIP is the a-arrestin family protein that is induced by oxidative stress and is known to inhibit the key antioxidant protein TRX via a direct interaction [15]. Previous studies showed that age-dependent upregulation of TXNIP resulted in decreased stress resistance to oxidative challenge in primary human cells and in Drosophila [25]. However, the mechanism in regulating TXNIP expression during cell senescence is unclearly known yet. Our previous study has already confirmed that miR-17 could directly target and suppress TXNIP expression. Thus, the abnormal redox status and the decreased TRX activity in senescent AMSCs may also due to the abrogated suppression of TXNIP by decreased miR-17. As expected, TXNIP expression was upregulated concomitant with the decreased miR-17 and miR-20a levels, the decreased TRX activity, and the enhanced ROS levels in senescent AMSCs. Moreover, TXNIP interference reversed miR-17 or miR-20a inhibitor transfection-induced TRX activity reduction and ROS elevation in AMSCs. These data further indicate that miR-17-92-mediated TXNIP and redox system regulation also play important role in AMSC senescence.

Finally, we confirmed that administration of miR-17- or miR-20a-modified AMSCs could more effectively attenuate LPS/GalN-induce ALF as compared to the administration of control miRNA-modified AMSCs. Besides its role in mediating oxidative stress, TXNIP is also known to mediate NLRP3 inflammasome activation. Our previous study showed that exosome-mediated miR-17 shuttling is involved in the therapeutic effects of AMSC-derived exosomes against ALF by targeting TXNIP and consequent NLRP3 inflammasome blockage [26]. Thus, the improved therapeutic efficacy of AMSCs by miR-17 or miR-20a modification might also due to the more effectively inhibition on NLRP3 inflammasome activation. Additionally, excessive ROS generation contribute to the occurrence and development of liver failure. Thus, the miR-17- or miR-20a-modified AMSCs may also more effectively suppressed ROS level by exosomal miRNA-mediated TXNIP-TRX intervention.

\section{Conclusions}

This study showed that the decreased level of miR-17-92 cluster, due to the reduced expression of c-Myc, was associated with the senescent features of AMSCs and the reduced therapeutic efficacy of AMSC on 
ALF. Thus, the cellular miR-17-92 cluster level, especially the miR-17 and miR-20a levels, can be used as an index to evaluate the therapeutic potential of AMSCs. Moreover, miR-17-92 cluster miRNAsmodification may provide a new strategy for reversing the cellular senescence and improving the therapeutic efficacy of AMSCs.

\section{Abbreviations}

MSC: mesenchymal stem cells; ALF: acute liver failure; oAMSCs: adipose tissue-derived MSCs from oldaged mice; yAMSC: AMSCs from young mice; LPS/GalN: lipopolysaccharide and D-galactosamine; SA- $\beta$ gal: senescence-associated $\beta$-galactosidase; SASP: senescence-associated secretory phenotype; ALT: alanine aminotransferase aspartate; AST: aspartate aminotransferase; TBiL: total bilirubin; ELISA: enzyme-linked immunosorbent assay; TNF-a: tumor necrosis factor-a; IL-6: Interleukin-6; MCP-1: Monocyte Chemoattractant Protein-1; oAMSC-M17: miR-17-modified oAMSCs; oAMSC-M20a: miR-20amodified oAMSCs; oAMSC-M67: control miRNA-modified oAMSCs; siRNA: small interfering RNA; M-Ctrl: miRNA mimic negative control; I-Ctrl: miRNA inhibitor negative control; TRX: Thioredoxin; TXNIP: thioredoxin-interacting protein; ROS: reactive oxygen species.

\section{Declarations}

\section{Ethics approval and consent to participate}

Animal experimental protocols were approved by the Institutional Animal Care and Use Committee of the First Affiliated Hospital of Zhejiang University.

\section{Consent for publication}

Not applicable.

\section{Availability of data and materials}

All data and materials are available upon request.

\section{Competing Interests}

The authors have declared that no competing interest exists.

\section{Fundings}

This study was supported by the National Natural Science Foundation of China (81870428), the Key R \& D Projects of Zhejiang Province (2018C03019) and the Independent Task of State Key Laboratory for Diagnosis and Treatment of Infectious Diseases.

\section{Author Contributions}


Yelei Cen and Jinjin Qi performed the in vivo and in vitro experiments and drafted the manuscript. Liang Chen participated in the in vitro studies and performed the statistical analysis. Yanning Liu and Guohua Lou conceived of the study and participated in its design and coordination. All the authors have read and approved the final manuscript.

\section{Acknowledgements}

Not applicable.

\section{References}

1. Stravitz RT, Lee WM. Acute liver failure. Lancet. 2019;394(10201):869-881.

2. Patel P, Okoronkwo N, Pyrsopoulos NT. Future approaches and therapeutic modalities for acute liver failure. Clin Liver Dis. 2018;22(2):419-427.

3. Saleh M, Taher M, Sohrabpour AA, Vaezi AA, Nasiri Toosi M, Kavianpour M, Ghazvinian Z, Abdolahi S, Verdi J. Perspective of placenta derived mesenchymal stem cells in acute liver failure. Cell Biosci. 2020;10:71.

4. Lou G, Yang Y, Liu F, Ye B, Chen Z, Zheng M, Liu Y. MiR-122 modification enhances the therapeutic efficacy of adipose tissue-derived mesenchymal stem cells against liver fibrosis. J Cell Mol Med. 2017;21(11):2963-2973.

5. Lou G, Chen L, Xia C, Wang W, Qi J, Li A, Zhao L, Chen Z, Zheng M, Liu Y. MiR-199a-modified exosomes from adipose tissue-derived mesenchymal stem cells improve hepatocellular carcinoma chemosensitivity through mTOR pathway. J Exp Clin Cancer Res. 2020;39(1):4.

6. Sepúlveda JC, Tomé M, Fernández ME, Delgado M, Campisi J, Bernad A, González MA. Cell senescence abrogates the therapeutic potential of human mesenchymal stem cells in the lethal endotoxemia model. Stem Cells. 2014;32 (7):1865-1877.

7. Kornicka K, Marycz K, Tomaszewski KA, Marędziak M, Śmieszek A. The Effect of Age on Osteogenic and Adipogenic Differentiation Potential of Human Adipose Derived Stromal Stem Cells (hASCs) and the Impact of Stress Factors in the Course of the Differentiation Process. Oxid Med Cell Longev. 2015;2015:309169.

8. Horiguchi M, Hata S, Tsurudome Y, Ushijima K. Characterizing the degeneration of nuclear membrane and mitochondria of adipose-derived mesenchymal stem cells from patients with type II diabetes. J Cell Mol Med. 2021;25(9):4298-4306.

9. Gnani D, Crippa S, Della Volpe L, Rossella V, Conti A, Lettera E, Rivis S, Ometti M, Fraschini G, Bernardo ME, Di Micco R. An early-senescence state in aged mesenchymal stromal cells contributes to hematopoietic stem and progenitor cell clonogenic impairment through the activation of a proinflammatory program. Aging Cell. 2019;18(3):e12933.

10. Dimmeler S, Nicotera P. MicroRNAs in age-related diseases. EMBO Mol Med. 2013;5(2):180-190. 
11. Auler M, Bergmeier V, Georgieva VS, Pitzler L, Frie C, Nüchel J, Eckes B, Hinz B, Brachvogel B. miR127-3p Is an Epigenetic Activator of Myofibroblast Senescence Situated within the MicroRNAEnriched DIk1-Dio3-Imprinted Domain on Mouse Chromosome 12. J Invest Dermatol. 2021;141(4S):1076-1086.e3.

12. Urtasun R, Elizalde M, Azkona M, Latasa MU, García-Irigoyen O, Uriarte I, Fernández-Barrena MG, Vicent S, Alonso MM, Muntané J, Prieto J, Ávila MA, Berasain C. Splicing regulator SLU7 preserves survival of hepatocellular carcinoma cells and other solid tumors via oncogenic miR-17-92 cluster expression. Oncogene. 2016;35(36):4719-4729.

13. Vaira V, Gaudioso G, Laginestra MA, Terrasi A, Agostinelli C, Bosari S, Di Sabatino A, Vanoli A, Paulli M, Ferrero S, Roncoroni L, Lombardo V, Perera LP, Fabris S, Vecchi M, Pileri S, Elli L. Deregulation of miRNAs-cMYC circuits is a key event in refractory celiac disease type-2 lymphomagenesis. Clin Sci (Lond). 2020;134(10):1151-1166.

14. Vono R, Jover Garcia E, Spinetti G, Madeddu P. Oxidative Stress in Mesenchymal Stem Cell Senescence: Regulation by Coding and Noncoding RNAs. Antioxid Redox Signal. 2018;29(9):864879.

15. Balsera M, Buchanan BB. Evolution of the thioredoxin system as a step enabling adaptation to oxidative stress. Free Radic Biol Med. 2019;140:28-35.

16. Block TJ, Marinkovic M, Tran ON, Gonzalez AO, Marshall A, Dean DD, Chen XD. Restoring the quantity and quality of elderly human mesenchymal stem cells for autologous cell-based therapies. Stem Cell Res Ther. 2017;8(1):239.

17. Lee JH, Yoon YM, Song KH, Noh H, Lee SH. Melatonin suppresses senescence-derived mitochondrial dysfunction in mesenchymal stem cells via the HSPA1L-mitophagy pathway. Aging Cell. 2020;19(3):e13111.

18. Ma X, Zheng Q, Zhao G, Yuan W, Liu W. Regulation of cellular senescence by microRNAs. Mech Ageing Dev. 2020 Jul;189:111264.

19. Kim YJ, Hwang SH, Lee SY, Shin KK, Cho HH, Bae YC, Jung JS. miR-486-5p induces replicative senescence of human adipose tissue-derived mesenchymal stem cells and its expression is controlled by high glucose. Stem Cells Dev. 2012;21(10):1749-1760.

20. Tomé M, Sepúlveda JC, Delgado M, Andrades JA, Campisi J, González MA, Bernad A. miR-335 correlates with senescence/aging in human mesenchymal stem cells and inhibits their therapeutic actions through inhibition of AP-1 activity. Stem Cells. 2014;32(8):2229-2244.

21. Mogilyansky E, Rigoutsos I. The miR-17/92 cluster: a comprehensive update on its genomics, genetics, functions and increasingly important and numerous roles in health and disease. Cell Death Differ. 2013;20(12):1603-1614.

22. Li Y, Choi PS, Casey SC, Dill DL, Felsher DW. MYC through miR-17-92 suppresses specific target genes to maintain survival, autonomous proliferation, and a neoplastic state. Cancer Cell. 2014;26(2):262-272. 
23. Liu W, Qi M, Konermann A, Zhang L, Jin F, Jin Y. The p53/miR-17/Smurf1 pathway mediates skeletal deformities in an age-related model via inhibiting the function of mesenchymal stem cells. Aging (Albany NY). 2015;7(3):205-218.

24. Zhuang Y, Li T, Xiao H, Wu J, Su S, Dong X, Hu X, Hua Q, Liu J, Shang W, Ju J, Sun F, Pan Z, Lu Y, Zhang M. LncRNA-H19 Drives Cardiomyocyte Senescence by Targeting miR-19a/socs1/p53 Axis. Front Pharmacol. 2021;12:631835.

25. Oberacker T, Bajorat J, Ziola S, Schroeder A, Röth D, Kastl L, Edgar BA, Wagner W, Gülow K, Krammer $\mathrm{PH}$. Enhanced expression of thioredoxin-interacting-protein regulates oxidative DNA damage and aging. FEBS Lett. 2018;592(13):2297-2307.

26. Liu Y, Lou G, Li A, Zhang T, Qi J, Ye D, Zheng M, Chen Z. AMSC-derived exosomes alleviate lipopolysaccharide/d-galactosamine-induced acute liver failure by miR-17-mediated reduction of TXNIP/NLRP3 inflammasome activation in macrophages. EBioMedicine. 2018;36:140-150.

\section{Figures}
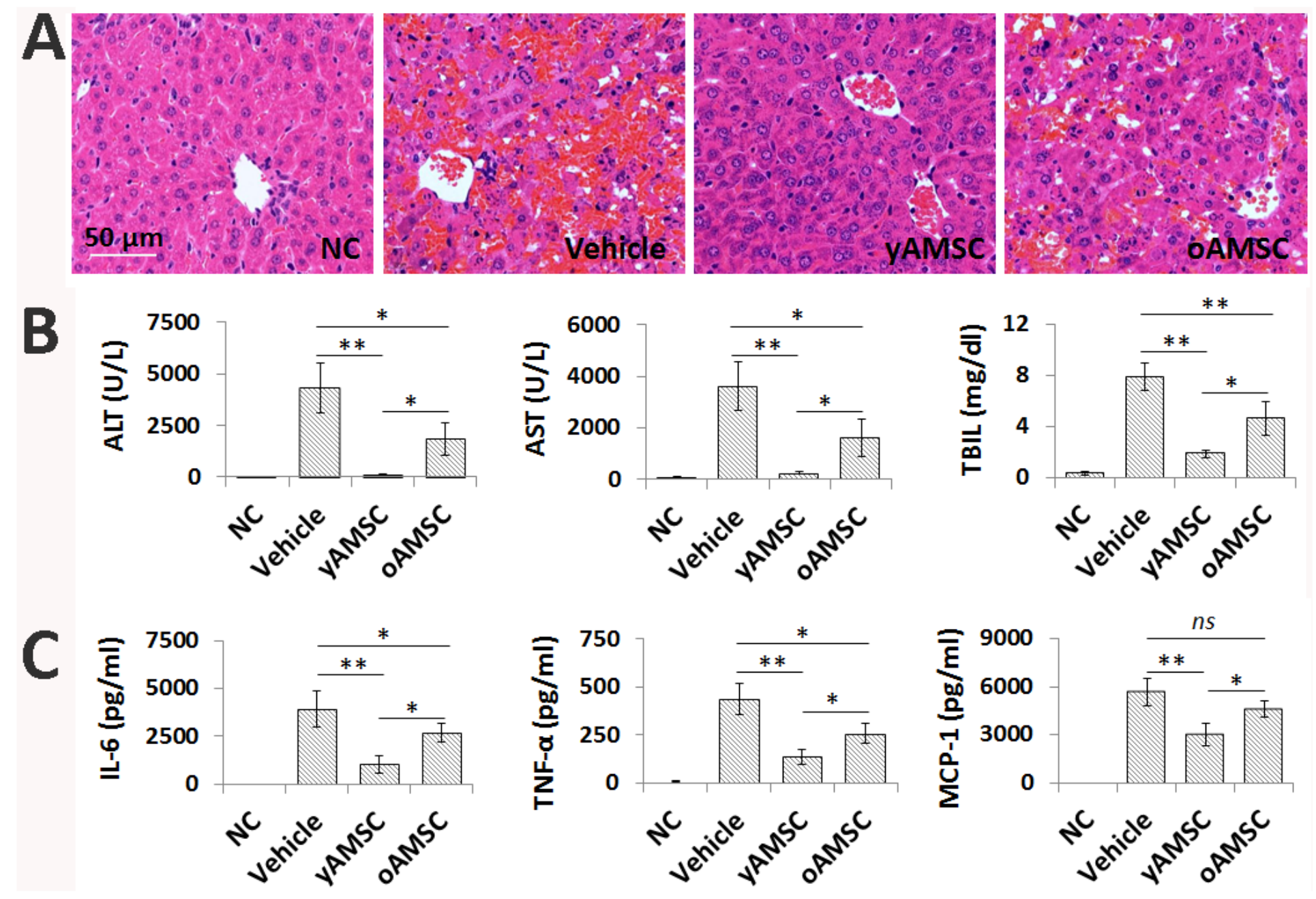

Figure 1 
Administration of AMSCs from aged mice has reduced therapeutic efficiency on LPS/GalN-induced liver failure

(A) H\&E staining of the pathological changes in the liver tissue. (B) Serum levels of ALT, AST, and TBIL in groups of normal control (NC), LPS/GalN-induced liver failure (vehicle group), and treatment with AMSCs from young mice (yAMSC) or AMSCs from old-aged mice (OAMSC). (C) The serum levels of inflammatory cytokines were determined by ELISA. Data are presented as the mean $\pm \mathrm{SD}(\mathrm{n}=6)$. ${ }^{\star} P<0.05 ;{ }^{\star \star} P<0.01$; $n s$, not significant.
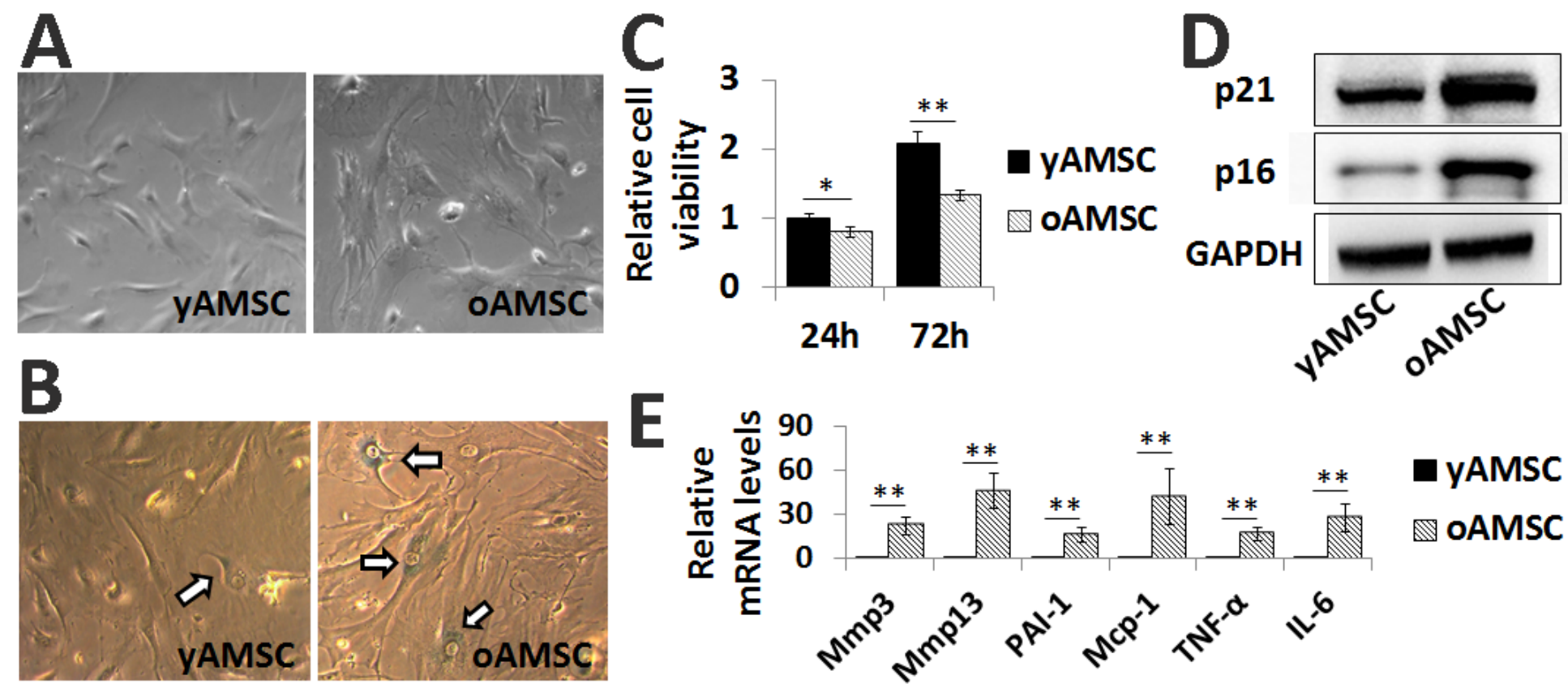

yAMSC $\mathbb{Q}$ OAMSC

Figure 2

Characterization of the senescence-associated features of AMSCs

(A) The morphology of AMSCs observed under an inverted microscope. (B) SA- $\beta$-gal staining (blue) of AMSCs observed under an inverted microscope. (C) The proliferation capacity of AMSCs was determined by CCK-8 assay. (D) Western blot analysis showed the expression levels of p16 and p21 in AMSCs. (E) The mRNA levels of SASP markers including Mmp3, Mmp13, PAl-1, MCP-1, TNF- , and IL-6 in AMSCs were analyzed by qPCR. Data are presented as the mean \pm SD $(n=3) .{ }^{*} P<0.05 ;{ }^{\star} P<0.01$; yAMSC, AMSCs from young mice; oAMSC, AMSCs from old-aged mice. 
A

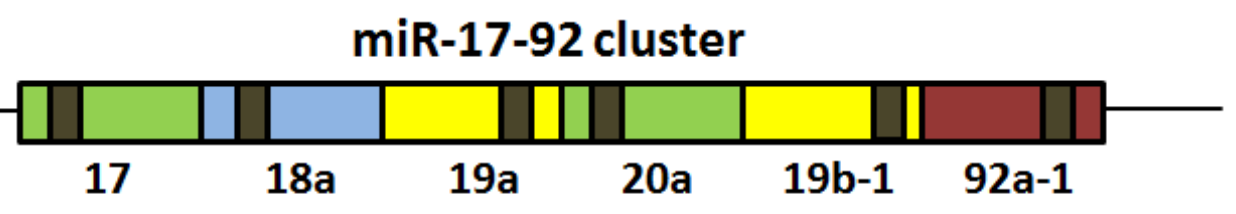

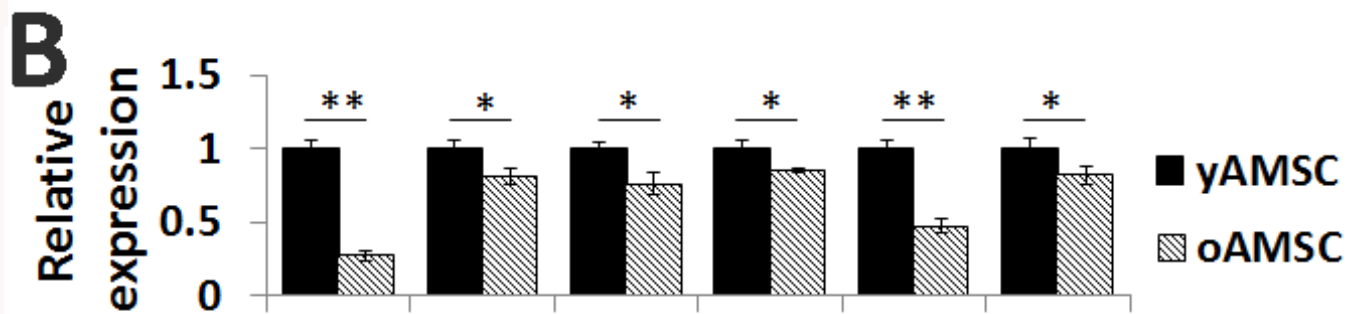

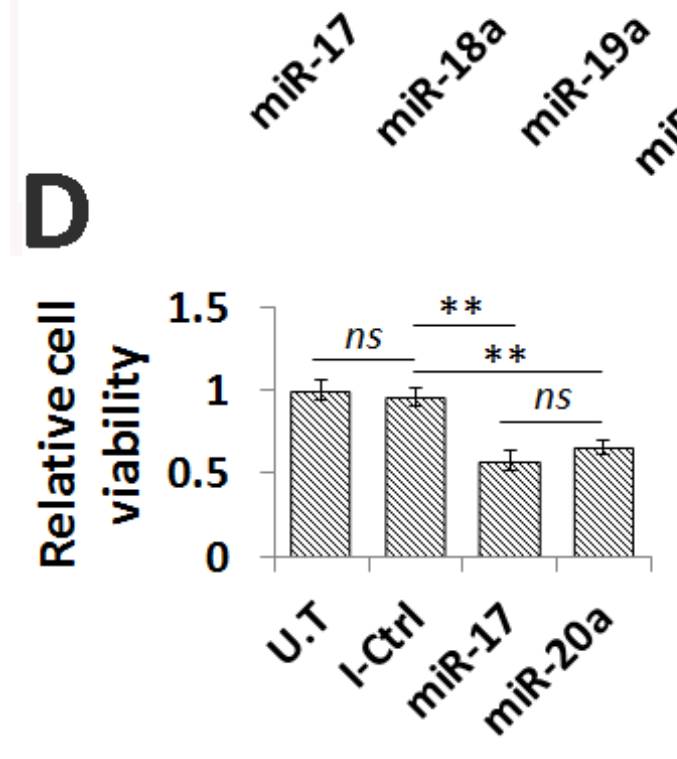

도

Target region

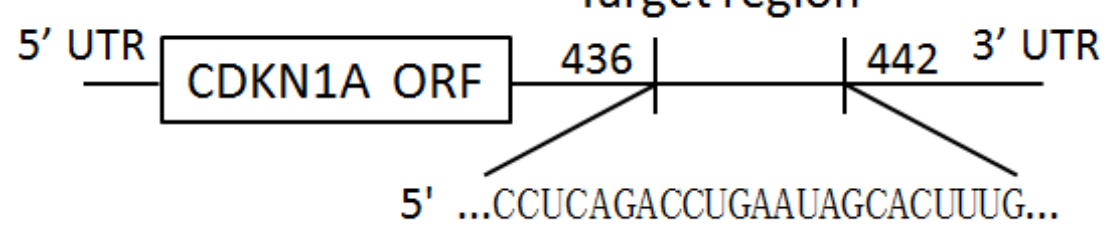

miR-17

IIIIIII

3' GAUGGACGUGACAUUUCGUGAAAC

5' ...CCUCAGACCUGAAUAGCACUUUG...

miR-20a

IIIIII

3' GAUGGaCGUGaUAUUUCGUGAAAU
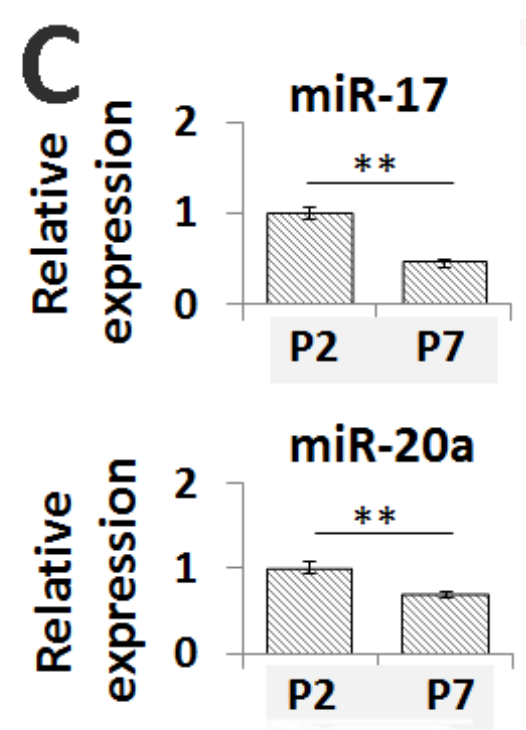
AMSCs transfected by the miR-17 and miR-20a inhibitors or the negative control inhibitor (I-Ctrl) at $72 \mathrm{~h}$ after transfection. (E) SA- $\beta$-gal staining (blue) of AMSCs transfected by miRNA inhibitors. (F) The diagram of the target region of miR-17 and miR-20a in the 3' UTR of CDKN1A, the gene encoding p21. (G) Western blot analysis showed the expression level of p21 in AMSCs transfected with miR-17 and miR-20a mimics or the negative control mimic (M-Ctrl). $(\mathrm{H})$ Western blot analysis of the p21 level in AMSCs transfected with miRNA inhibitors. Data are presented as the mean \pm SD $(n=3) . * * P<0.01$; yAMSC, AMSCs from young mice; OAMSC, AMSCs from old-aged mice.

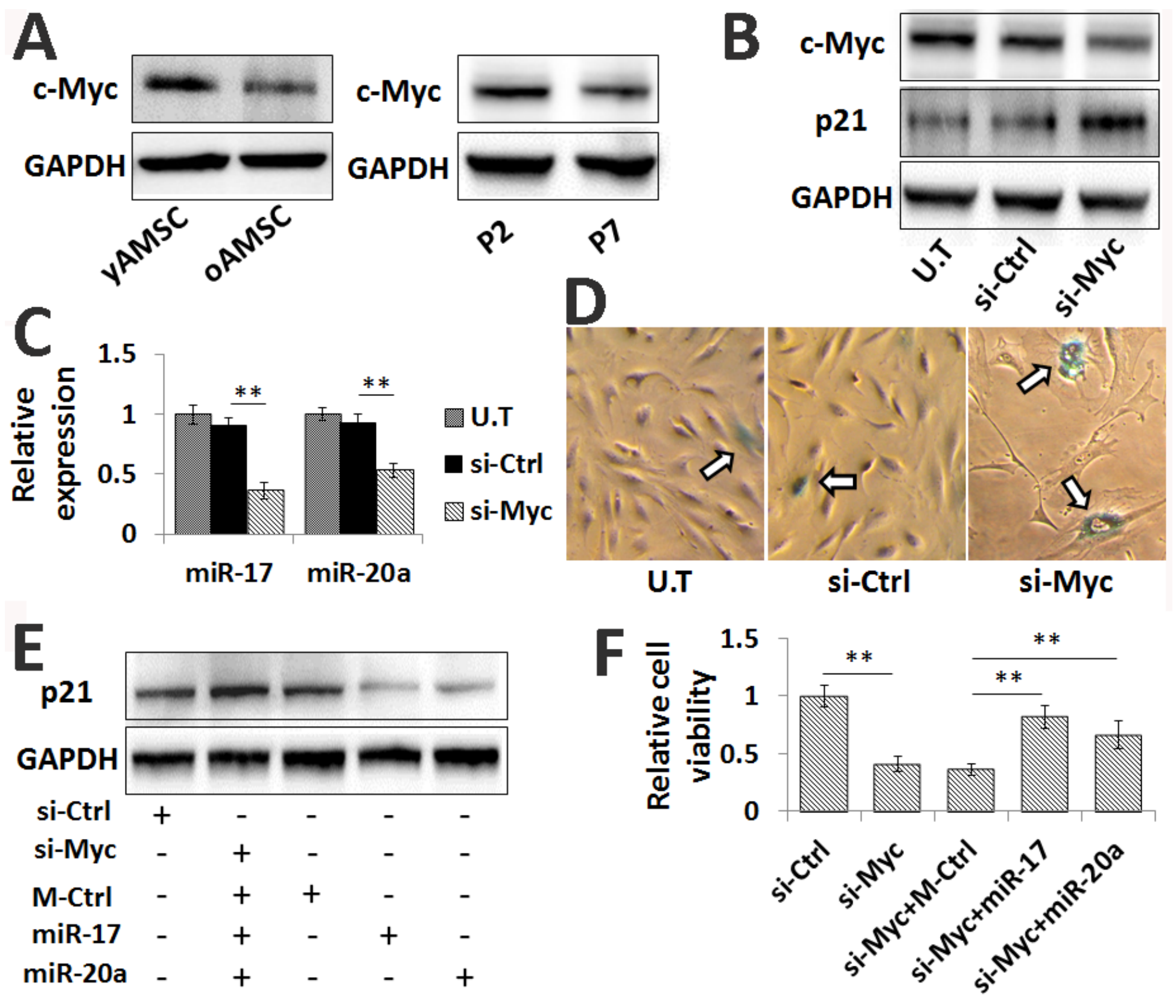

Figure 4

MiR-17-92 cluster is regulated by c-Myc during AMSC senescence

(A) Western blot analysis of the c-Myc level in AMSCs from young mice (yAMSC) or old-aged mice (OAMSC) and in a culture-induced senescent cell model of AMSCs. (B) Western blot analysis of the c-Myc 
and p21 levels in AMSCs following the transfection with the siRNA against c-Myc (si-Myc) or the scrambled siRNA (si-Ctrl). (C) qPCR analysis of the relative expression levels of miR-17 and miR-20a in AMSCs following the transfection with the above siRNA. (D) SA- $\beta$-gal staining (blue) of AMSCs transfected by the above siRNA. (E) Western blot analysis on p21 expression levels in AMSCs transfected by si-Myc, si-Ctrl or co-transfected by si-Myc and miRNA mimics at $48 \mathrm{~h}$ after transfection. (F) CCK-8 assay showed the proliferation capacity of AMSCs transfected by the above siRNA and mimics at $72 \mathrm{~h}$ after transfection. Data are presented as the mean $\pm S D(n=3) .{ }^{\star} * P<0.01 ; P 2$, AMSCs at passage 2; P7, AMSCs at passage 7; M-Ctrl, negative control mimic; U.T, untreated AMSCs.

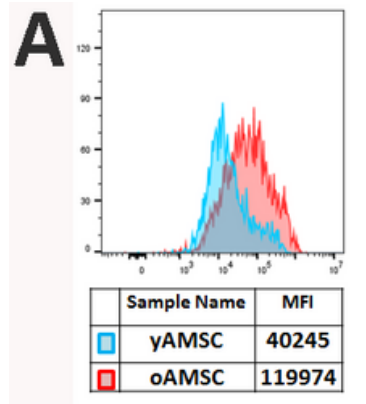

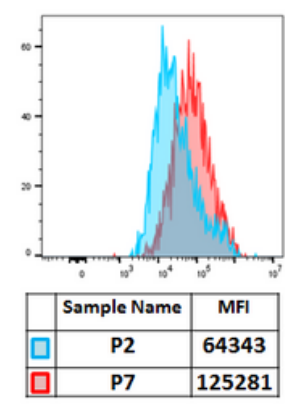

Target region

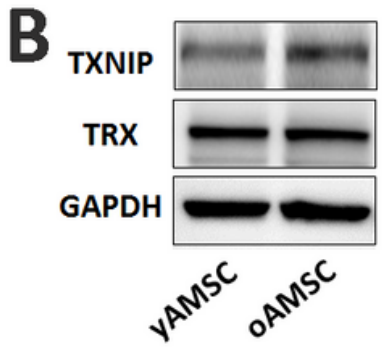

Target region

D

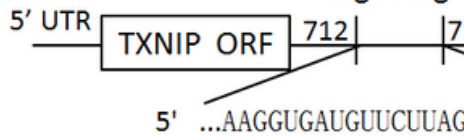

miR-17

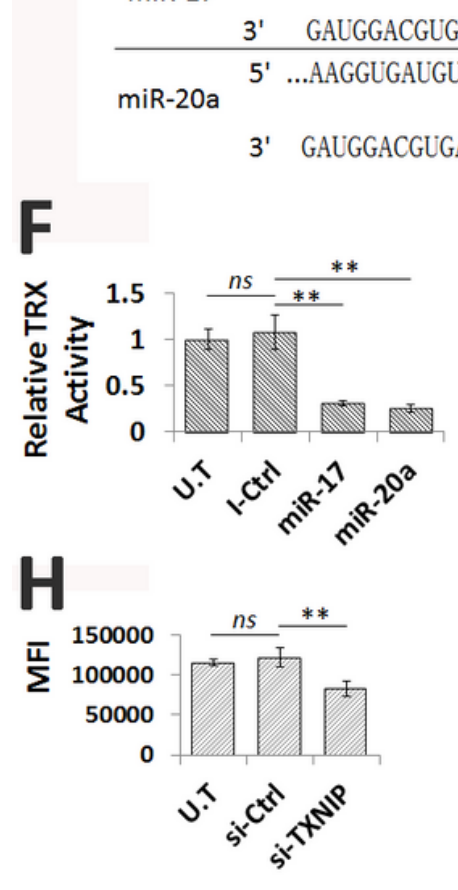

$\mathbf{H}$
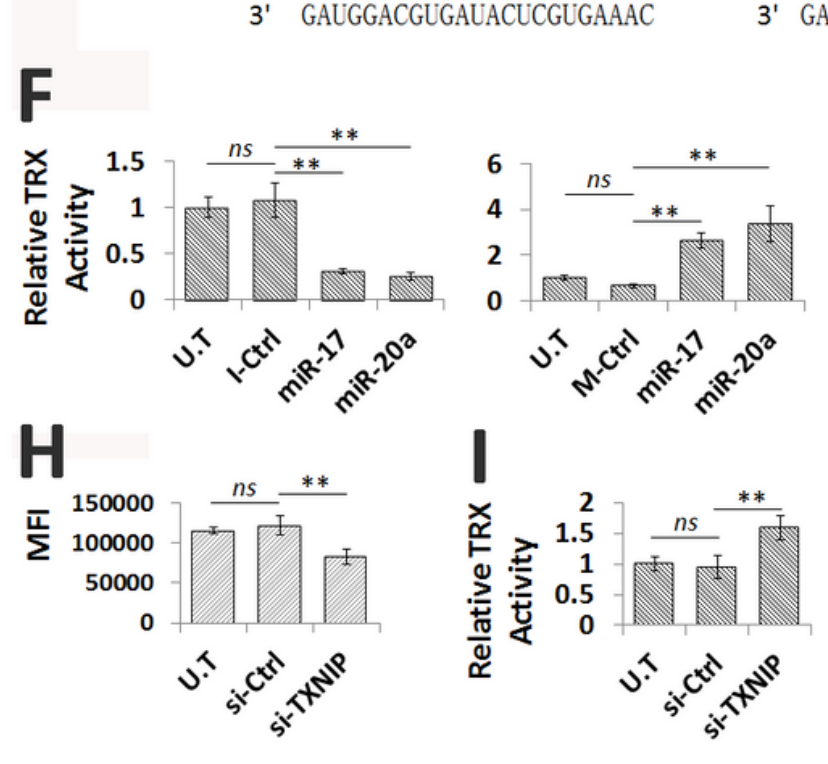

| || ||

IIIII

||||||

CGUGAAAC

Figure 5

Decreased miR-17-92 cluster associates with the disrupted oxidative homeostasis in senescent AMSCs via TXNIP regulation

(A) Flow cytometry analysis for ROS level in healthy AMSCs (yAMSC and P2) and senescent AMSCs (oAMSC, P7). (B) Western blot analysis on TRX and TXNIP expression levels in AMSCs. (C) Relative TRX 
activity was determined by setting the TRX fluorometric activity of healthy AMSCs (yAMSC and P2) as 1. (D) The diagram of the target region of miR-17 and miR-20a in the 3' UTR of TXNIP. (E) Western blot analysis on TXNIP level in AMSCs transfected with miRNA inhibitors (up) or miRNA mimics (down). (F) Relative TRX activity was determined by setting the TRX fluorometric activity of non-transfected (untreated) AMSCs as 1. (G) Western blot analysis on TXNIP level in AMSCs following the transfection with the siRNA against TXNIP (si-TXNIP) or the scrambled siRNA (si-Ctrl). (H) Mean fluorescence intensity (MFI) of ROS level in AMSCs following siRNA transfection. (I) Relative TRX activity was determined by setting the TRX fluorometric activity of non-transfected (untreated) AMSCs as 1. ( $\mathrm{J}$ and K) The relative TRX activity $(\mathrm{J})$ and the MFI of ROS level $(\mathrm{K})$ in miR-17 or miR-20a inhibitors-transfected AMSCs combined with si-TXNIP transfection. Data are presented as the mean $\pm \mathrm{SD}(\mathrm{n}=3) .{ }^{\star} P<0.05 ; \star \star P<0.01$; $n s$, not significant; P2, AMSCs at passage 2; P7, AMSCs at passage 7; I-Ctrl, negative control inhibitor; $M$ Ctrl, negative control mimic; U.T, untreated AMSCs. 


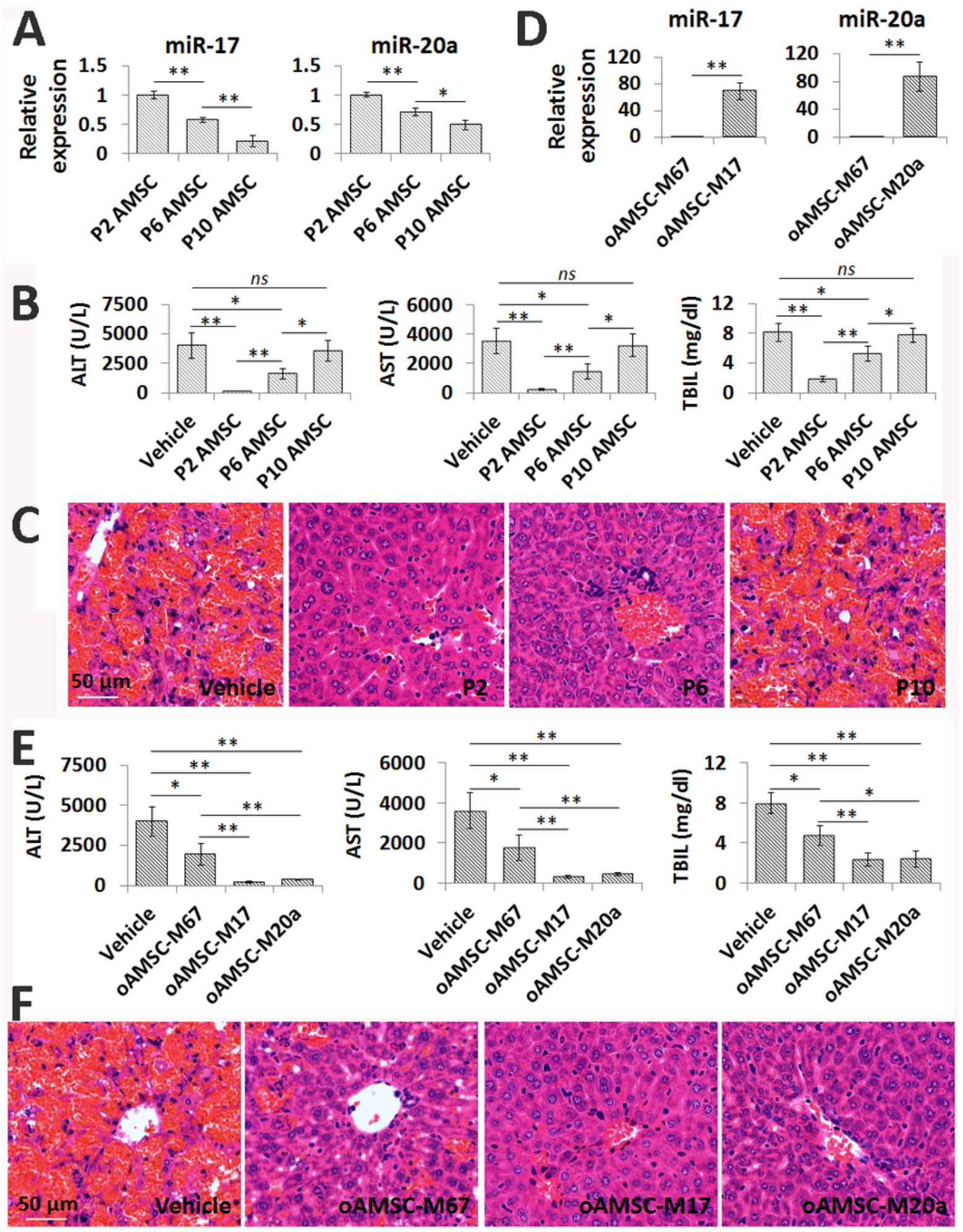

Figure 6

The therapeutic efficacy of AMSCs on acute liver failure is correlated with cellular miR-17-92 level

(A) qPCR analysis of the relative expression levels of miR-17 and miR-20a in AMSCs at passage 2 (P2), 6 (P6), and 10 (P10). (B) Serum levels of ALT, AST, and TBIL of the mice model of LPS/GalN-induced liver failure by vehicle or AMSC treatment. (C) H\&E staining of the pathological changes in the liver tissue of 
mice by vehicle or AMSC treatment. (D) qPCR analysis of the relative expression levels of miR-17 and miR-20a in miR-17- or miR-20a-modified AMSCs, which was constructed by infection of LV-miR-17 (oAMSC-M17) and LV-miR-20a into oAMSCs (oAMSC-M20a). oAMSCs infection with LV-cel-miR-67 was served as control (OAMSC-M67). (E and F) Serum levels of ALT, AST, and TBIL (E) and H\&E staining of liver sections $(F)$ of mice by vehicle or miRNA modified-AMSC treatment. Data are presented as the mean \pm SD $(n=6) .{ }^{\star} P<0.05 ;{ }^{\star} P<0.01 ; n s$, not significant; oAMSC, AMSCs from old-aged mice. 